Article

\title{
A Mutation in Mtap2 Is Associated with Arrest of Mammalian Spermatocytes before the First Meiotic Division
}

\author{
Fengyun Sun and Mary Ann Handel * \\ The Jackson Laboratory, 600 Main Street, Bar Harbor, ME 04609, USA; \\ E-Mail: fengyun.sun@jax.org \\ * Author to whom correspondence should be addressed; E-Mail: maryann.handel@jax.org; \\ Tel.: +1-207-288-6778; Fax: +1-207-288-6073.
}

Received: 25 October 2010; in revised form: 7 December 2010 / Accepted: 15 December 2010 / Published: 10 January 2011

\begin{abstract}
In spite of evolutionary conservation of meiosis, many of the genes that control mammalian meiosis are still unknown. We report here that the ENU-induced repro4 mutation, identified in a screen to uncover genes that control mouse meiosis, causes failure of spermatocytes to exit meiotic prophase I via the G2/MI transition. Major events of meiotic prophase I occurred normally in affected spermatocytes and known regulators of the meiotic G2/MI transition were present and functional. Deep sequencing of mutant DNA revealed a mutation located in an intron of the Mtap2 gene, encoding microtubule-associated protein 2, and levels of Mtap2 transcript were reduced in mutant testes. This evidence implicates MTAP2 as required directly or indirectly for completion of meiosis and normal spermatogenesis in mammals.
\end{abstract}

Keywords: meiosis; spermatogenesis; microtubule-associated protein

\section{Introduction}

In mammals, meiosis is comprised of two consecutive cell divisions following one round of DNA replication to give rise to four haploid secondary spermatocytes. In spite of evolutionary conservation of the process, many of the genes that control mammalian meiosis are still unknown and the degree of sexual dimorphism in meiosis remains a puzzle [1]. Identification of genes involved in meiosis can provide insight for better understanding of "maturation arrest" male infertility phenotypes. In humans, 
about half of infertility is related to male reproductive function; however, the etiology of much of this is not known [2-4].

Our work focuses on one of the least understood steps in meiosis I, which is the exit from the extended prophase I and onset of the division phase, also known as the meiotic prophase I to metaphase I, or G2/MI, transition. This is regulated in part by MPF (metaphase-promoting factor), comprised of a catalytic subunit, cyclin dependent kinase 1 (CDK1), and a regulatory subunit, cyclin B1 (CCNB1) [5-7]. The association of CDK1 with CCNB1 represents a key step and is regulated by the chaperone protein HSPA2 [7-9]. Together, these and other as yet unknown regulators bring about progressive changes in the nucleus and cytoskeleton, including desynapsis, chromosome condensation, disassembly of the nucleolus, and nuclear envelope breakdown [5,10-12]. Consequently, it is believed that the cytoskeleton may play roles in these events, and, interestingly, cytoskeletal elements, such as actin [13,14] and microtubule-associated proteins (MAPs) [15-17] have been reported in nuclei of spermatocytes. Furthermore, MAPs have been shown to mediate the association of MPF with microtubules [18,19]. Thus, function of microtubules and MAPs in meiotic cell cycle events is possible, but there has not been genetic evidence that this is the case.

Here we used an unbiased forward genetics strategy [20-22] to produce phenotypes of meiotic arrest and aberrant exit from meiotic prophase I in mouse spermatocytes. These phenotypes were found in homozygous repro4/repro4 mutants (here designated as repro4 mutants). A mutation in an intron of the Mtap2 gene, encoding microtubule-associated protein 2 protein, MTAP2, was identified in repro4 mutant DNA. Levels of the Mtap2 transcript are reduced in repro4 mutant testes. The meiosis arrest phenotype associated with the Mtap2 mutation in repro4 mutant mice provides the first genetic evidence that a microtubule-associated protein functions, either directly or indirectly, in meiotic prophase I exit during mammalian spermatogenesis.

\section{Results and Discussion}

The repro4 mutant line was produced by the NIH-funded Reproductive Genomics program at The Jackson Laboratory [22]. The mutation was induced on a C57BL/6J (herein, B6) background and ENU-treated males were subsequently mated to $\mathrm{C} 3 \mathrm{HeB} / \mathrm{FeJ}$ (herein, $\mathrm{C} 3 \mathrm{H}$ ) females to generate $\mathrm{G} 1$ males, which were mated with $\mathrm{C} 3 \mathrm{H}$ females to produce $\mathrm{G} 2$ offspring. $\mathrm{G} 2$ females were backcrossed to their G1 fathers to generate G3 progeny, which were tested for fertility and used to generate mice for initial gene mapping. The repro4 mutation segregates as a simple recessive Mendelian trait causing male-limited infertility.

\subsection{The repro4 Mutation Causes Meiotic Prophase I Arrest in Males}

The repro4 mutant males were overtly normal except that testis weights of adult mutants were about one third that of wild type (WT) littermates (Table 1) and only a few epididymal sperm were recovered from adult repro4 mutants. Nonetheless, the repro4 mutant males produced plugs in female mice, suggesting normal mating behavior. 
Table 1. Mean testis weights of WT and repro4 mutant mice.

\begin{tabular}{ccccc}
\hline Genotype & $\boldsymbol{n}$ & Age (weeks) & Body weight $(\mathbf{g})$ & Testis weight $(\mathbf{m g})$ \\
\hline WT & 5 & 8 & $27.18 \pm 1.30$ & $84.18 \pm 2.10$ \\
\hline repro4 mutant & 7 & 8 & $28.7 \pm 2.67$ & $35.45 \pm 1.52$ \\
\hline
\end{tabular}

Data are presented as mean \pm SEM.

In adult WT males, all stages of spermatogenesis-spermatogonia, spermatocytes, and spermatids - were observed in seminiferous tubules (Figure 1a). However in repro4 mutants, although spermatogonia and spermatocytes were observed, only a few tubules with spermatids were observed (Figure 1b), suggesting arrest of spermatogenesis in prophase of meiosis I. To determine whether the mutation influences spermatogonial proliferation, antibody to GCNA1 (germ cell nuclear antigen1) [23] was used to label the testis sections from postnatal day 8, 12, 17 mice; there were no differences in the number of spermatogonia between the mutants and their WT littermates (data not shown). Together, these observations reveal that the repro4 mutation does not severely impact spermatogonial proliferation, but causes arrest of spermatogenesis during prophase I.

To more precisely define the stage and nature of the spermatogenesis arrest phenotype of the repro 4 mutation, we analyzed the first wave spermatogenesis in detail by histology and immunofluorescent detection of markers of meiotic progression. Histology of testes at postnatal day 8 (P8), P11 and P14 showed no obvious differences in either morphology or histological relationships of various cell types or germ cell number between repro4 mutants and WT littermates (data not shown). By P17, spermatogonia and spermatocytes were observed in both WT and repro4 mutant testes. However, more spermatocytes with condensed chromatin, an indication of apoptosis, were observed in repro4 mutant testes than in testes of WT littermates (Figure 1c and d, arrow and inset in d). By P24, in marked contrast to WT littermates (Figure 1e, arrow and inset), very few round spermatids were seen in repro4 mutant testes (Figure 1f). In repro4 mutant testes, germ cells in most seminiferous tubules were at stages of prophase I, although metaphase spermatocytes were observed in a few tubules (Figure 1f, arrow and inset). Thus, in repro4 mutant testes, progress of spermatogenesis appeared to be arrested during prophase of meiosis I, although a few spermatocytes reached more advanced stages.

These observations of histology sections suggested spermatocytes in repro4 mutants reached the pachytene substage of meiotic prophase I. To confirm this, surface-spread spermatocyte chromatin was analyzed for presence of histone H1t, a marker of the mid-pachytene stage. As shown in Figure 2a, b, histone H1t was detected with appropriate nuclear localization in repro4 mutant spermatocytes, confirming that germ cell development in repro4 mutants reaches at least the mid-pachytene stage before arrest. Thus, although repro4 mutant spermatocytes reach the stage when competence to undergo the meiotic prophase I to metaphase I transition is normally acquired [5,6], the repro4 mutant spermatocytes failed to undergo this transition successfully in vivo. 
Figure 1. Spermatogenesis is arrested predominantly during prophase of meiosis I in repro4 mutants. PAS-stained testis sections from repro4 mutants and WT littermate controls reveal that spermatogonia, spermatocytes and spermatids are present in testes of adult WT mice (a), whereas in repro4 mutant testes, most tubules contain only spermatogonia and spermatocytes (b). By P17, during the first wave of spermatogenesis, WT and repro4 mutant testis tubules were populated with spermatocytes, but repro4 mutants (d) exhibited more spermatocytes with highly condensed chromatin (arrow and inset) than did WT testes (c). By P24, round spermatids were present in WT testes (e, arrow and inset), but repro4 mutant testis tubules contained primarily prophase and a few metaphase spermatocytes (f, arrow and inset). Scale bar for $\mathbf{a}-\mathbf{f}=100 \mu \mathrm{m}$.

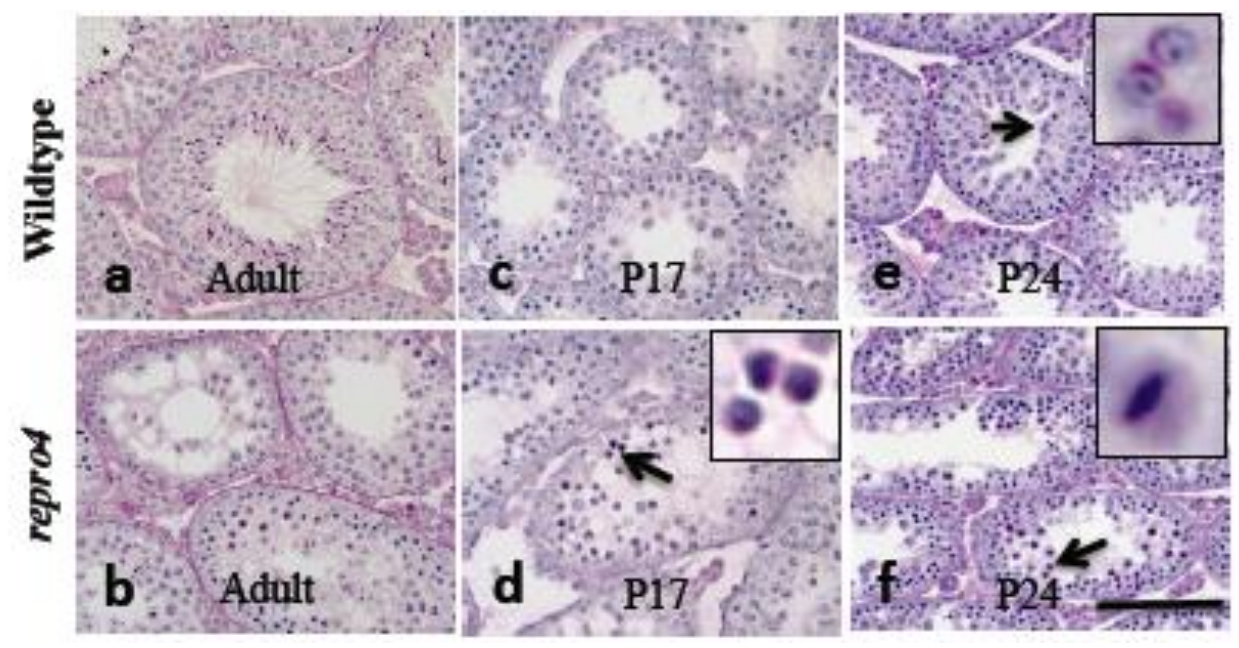

Because of this arrest, we assessed whether repro4 mutant spermatocytes undergo apoptosis. TUNEL analysis revealed a significant increase $(p<0.05)$ of the number of apoptotic cells per tubule in repro4 mutant testes compared to littermate controls (Table 2). An increase of the frequency of tubules with apoptotic cells in repro4 mutant testes was also observed compared to that in WT controls at $\mathrm{P} 17$, although the difference was not significant (Table 2). The apoptotic cells were detected luminal from the basement membrane of the seminiferous tubules, not apposed to the basement membrane. This location suggests that they were spermatocytes, but not spermatogonia or Sertoli cells. This inference was bolstered by GCNA staining, which revealed no difference in the number of spermatogonia between the repro4 mutant and WT testes (see above). Moreover, an increase in apoptosis was also observed by flow cytometry after labeling apoptotic cells with annexin V (data not shown). Thus germ cells in repro4 mutant testes undergo apoptosis during meiotic prophase I.

Table 2. Analysis of apoptosis in repro4 mutants.

\begin{tabular}{ccccc}
\hline & WT, P17 & repro4, P17 & WT, P21 & repro4, P21 \\
\hline Apoptotic tubules/total tubules & $0.34 \pm 0.05$ & $0.43 \pm 0.03$ & $0.38 \pm 0.02$ & $0.47 \pm 0.07$ \\
\hline Apoptotic cells/tubule & $0.69 \pm 0.11 *$ & $2.19 \pm 0.38 *$ & $0.85 \pm 0.13 *$ & $2.17 \pm 0.36 *$ \\
\hline
\end{tabular}

Data are presented as mean \pm SEM. $*, p<0.05$. P, postnatal day; WT, wild type; repro4, repro4 mutant. Three animals of each group and at least 40 tubules of each animal were analyzed. 


\subsection{Major Cytological Events of Prophase I in repro4 Mutants Are Apparently Normal}

To determine if the failure of repro4 mutant spermatocytes to enter the meiotic division phase was due to deficiencies of key events of meiotic prophase I, we assessed markers of these events, including synaptonemal complex formation, meiotic DNA double-strand break formation and repair, and localization of DNA damage checkpoint proteins. As shown in Figure 2c, d, the labeling patterns for SYCP3 and SYCP1 proteins, components of the lateral element and central element, respectively, of the synaptonemal complex, in repro4 mutant spermatocytes was indistinguishable from that in WT littermate spermatocytes. Thus synapsis appears to be substantially normal in repro4 mutant germ cells, and, indeed, even diplotene spermatocytes were found among repro4 mutant spermatocytes, as determined from the labeling pattern of SYCP3 (data not shown). During meiosis, DNA double strand breaks (DSBs) occur in early prophase and are then repaired as germ cells reach the pachytene stage. The localization of phosphorylated histone $\mathrm{H} 2 \mathrm{AFX}(\gamma \mathrm{H} 2 \mathrm{AX})$, a marker of DNA DSBs, was identical in leptonema/zygonema and pachynema of both repro4 mutants and WT littermates (Figure 2e-h), suggesting that as in WT spermatocytes, DSBs occurred and were repaired in the repro4 mutant spermatocytes. Ataxia telangiectasia and Rad3-related protein (ATR) is involved in sensing DNA damage and activating the DNA damage checkpoint, leading to cell cycle arrest [24]. To verify that DSBs were repaired in repro4 mutant spermatocytes and confirm that the arrest of repro4 mutant spermatocytes was not due to DNA damage, ATR labeling was performed. Consistent with $\gamma \mathrm{H} 2 \mathrm{AX}$ labeling, the labeling pattern of ATR was indistinguishable between repro4 mutant spermatocytes and littermate controls (Figure 2i, j). Likewise, condensation of the $\mathrm{X}$ and $\mathrm{Y}$ chromosomes to form the sex body occurred normally in repro4 mutant spermatocytes, as indicated by the accumulation of $\gamma \mathrm{H} 2 \mathrm{AX}$ in the sex body, marking the condensed X and Y chromosomes (Figure 2g, h).

Figure 2. Essential events in the prophase of meiosis I appear normal in repro4 mutant testes. By P17, both WT (a) and repro4 mutant (b) spermatocytes have accumulated histone H1t (green), a marker of the mid-pachytene stage, seen in the chromatin surrounding the SYCP3 axes (red) of the synaptonemal complexes (SC). The extent of synapsis, reflected by the patterns of SYCP1 (green), a central element of the SC, and SYCP3 (red), a lateral element of the SC, was indistinguishable between WT (c) and repro4 mutant (d) spermatocytes. The pattern of $\gamma \mathrm{H} 2 \mathrm{AX}$ labeling, a marker of DNA double strand break/repair, was also similar between WT (e and $\mathbf{g}$ ) and repro4 mutant (f and $\mathbf{h})$ spermatocytes in early prophase (e and $\mathbf{f}$ ) and in the mid-to late pachytene stage ( $\mathbf{g}$ and $\mathbf{h}$ ). The labeling pattern of the DNA damage checkpoint protein ATR at pachytene stage appears normal in repro4 mutant spermatocytes (j) compared to WT controls (i). Scale bar $=10 \mu \mathrm{m}$.

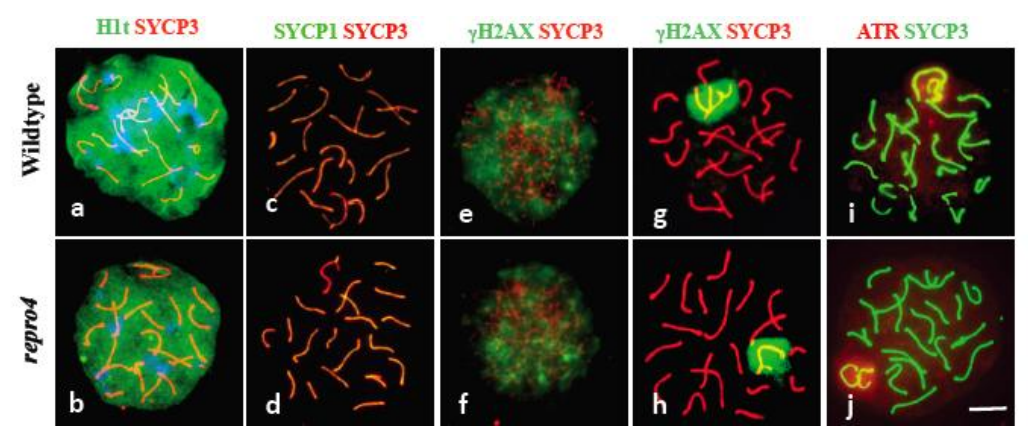


These observations suggest that DNA damage/repair, and meiotic chromosome pairing and synapsis in repro4 mutant germ cells are cytologically normal, and may not be causative for the failure of mutant spermatocytes to undergo the transition from meiotic prophase I to metaphase I. We thus hypothesized that the cell cycle machinery may not be functional in repro4 mutant spermatocytes. The universal regulator of prophase to metaphase transition in both mitosis and meiosis is MPF, composed of a regulatory subunit of CCNB1 and a catalytic subunit of CDK1 [6,25,26]. Immunohistochemistry and western blotting were used to assess the presence of CCNB1 and CDK1 in repro4 mutant spermatocytes. As shown in Figure 3, CDK1 and CCNB1 were observed in repro4 mutant spermatocytes as in WT spermatocytes.

Figure 3. Protein components of MPF are present in repro4 mutant spermatocytes. Both immunohistochemistry (a, b, d and e) and western blotting (c and f) revealed that CDK1 (a, b and $\mathbf{c}$ ) and $\mathrm{CCNB} 1$ (d, e and f) were present in both WT and repro4 mutant spermatocytes. WT, wild type; repro4, repro4 mutant. Scale bar $=100 \mu \mathrm{m}$.

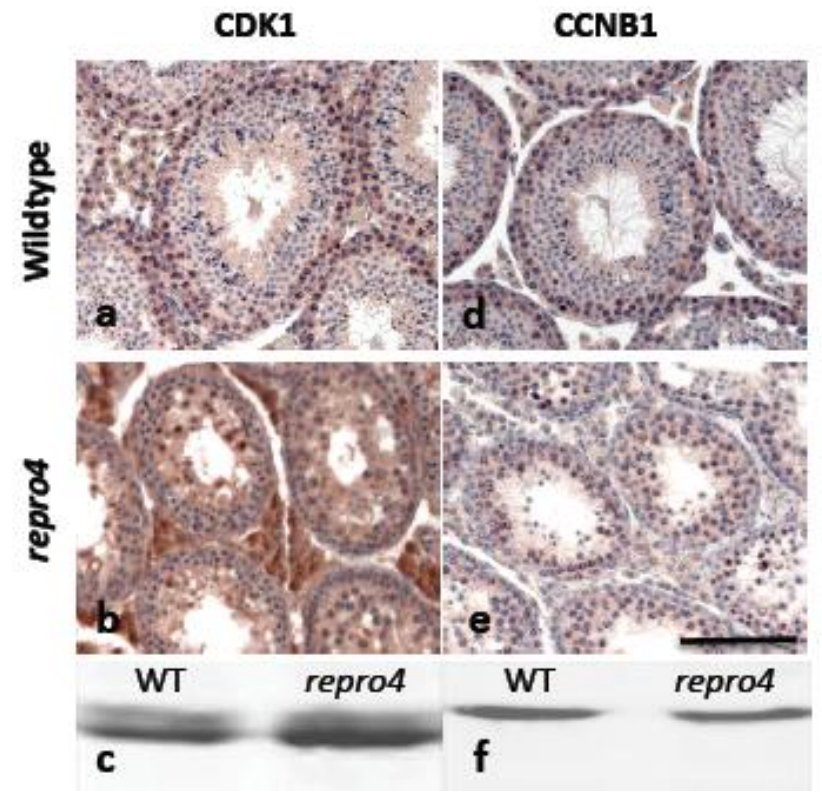

To determine competence of repro4 mutant spermatocytes to undergo the G2/MI transition, we treated repro4 mutant and WT spermatocytes in vitro with okadaic acid (OA), an inhibitor of protein phosphatase 1 and 2A. OA induces MPF activation and the meiotic prophase I to metaphase I transition in vitro [5]. Progress of the G2/MI transition was evaluated by two parameters: The formation of fully condensed and individualized metaphase I bivalent chromosomes and phosphorylation of histone $\mathrm{H} 3$. Histone $\mathrm{H} 3$ phosphorylation occurred in repro4 mutant spermatocytes as in WT littermate spermatocytes (Figure 4a, b), suggesting that aurora kinase B, reported to be involved in meiotic prophase I to metaphase I transition [6], was functional in the repro4 mutant spermatocytes. However, although chromatin condensation was observed in OA-treated repro4 mutant spermatocytes, unlike chromosomes of WT spermatocytes (Figure 4c), many repro4 mutant spermatocytes could not form individualized bivalent metaphase I chromosomes after OA treatment (Figure 4d). This suggests the failure of G2/MI chromatin remodeling events in repro4 mutant spermatocytes in vitro as well as in vivo. 
Figure 4. The OA-induced G2/MI transition is not normal in repro4 mutant spermatocytes. After treatment of spermatocytes with okadaic acid in vitro, phosphorylation of histone $\mathrm{H} 3$ (green) around the SYCP3 axes of the synaptonemal complex (red) occurred in both WT (a) and repro4 mutant (b) spermatocytes. (c) shows the normal chromosome configuration after spermatocytes were treated with $\mathrm{OA}$ in vitro, and (d) shows a representative chromosome configuration after repro4 mutant spermatocytes were treated with OA in vitro. (e) presents these observations graphically and demonstrates that about 65\% WT spermatocytes formed condensed and individualized bivalent chromosomes, whereas significantly fewer $(p<0.01$, about $30 \%$ ) repro4 mutant spermatocytes formed condensed and individualized bivalent chromosomes. This experiment was performed 3 times, with at least 200 spermatocytes counted per experiment. WT, -OA: wild type spermatocytes without OA treatment; WT, +OA: wild type spermatocytes with OA treatment; repro4, -OA: repro4 mutant spermatocytes without OA treatment; repro4, +OA: repro4 mutant spermatocytes with OA treatment. Scale bar for $\mathbf{a}-\mathbf{d}=10 \mu \mathrm{m}$.
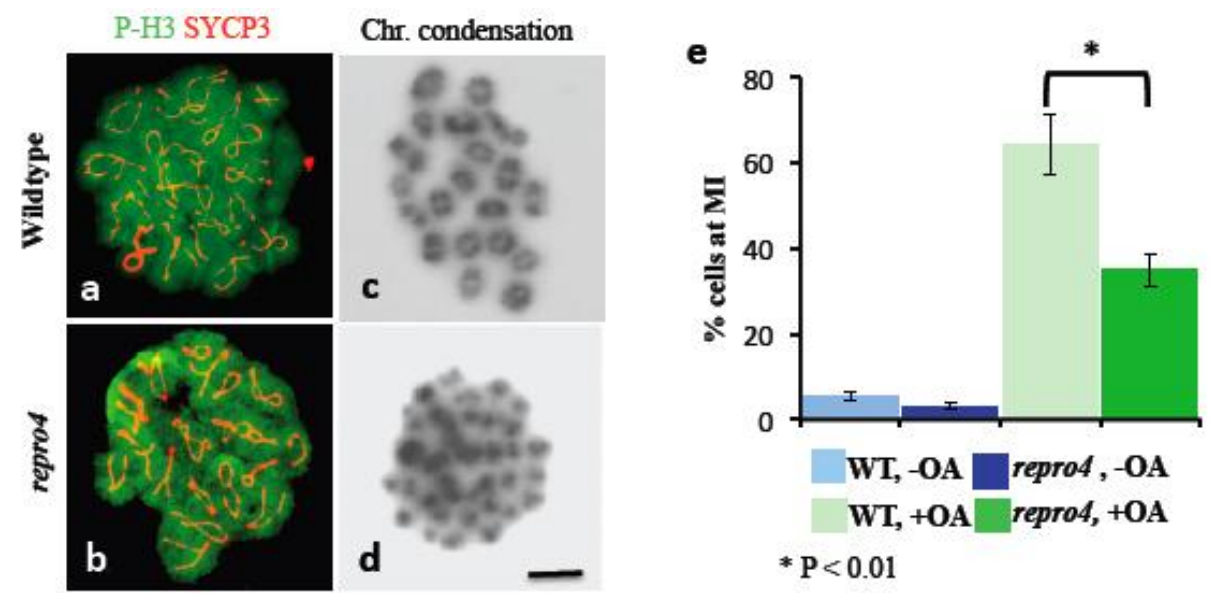

Taken together, the results presented in Figures 3 and 4 indicate that major events of prophase of meiosis I occur in repro4 mutant spermatocytes, and at least some of the cell cycle machinery required for the G2/MI transition is functional. However, repro4 mutant spermatocytes are incapable of executing the meiotic G2/MI transition normally in vivo and in vitro. These observations suggest that some as yet unknown factor must be required for regulation of this transition and chromatin remodeling and be absent or defective in repro4 mutant testes.

\subsection{Sequencing of repro4 Mutant DNA Reveals a T to A Transversion Mutation in Mtap2}

A genome scan of DNA from affected and unaffected mice in the repro4 family for polymorphic markers on each autosomal chromosome revealed the repro4 mutation on Chr 1 between D1Mit303 and D1Mit415, a region of about $25.4 \mathrm{Mb}$. Fine mapping within this region was conducted. Homozygous repro4 mutant females were crossed to CAST/EiJ (herein CAST) males, and the F1 offspring were intercrossed to produce F2 individuals that were subjected to phenotype analysis and typed for polymorphic markers within the region. Additionally, recombinants obtained from the $\mathrm{C} 3 \mathrm{H}$-repro4 maintenance line were also used to narrow the candidate region. The critical region for 
the repro4 mutation was an interval of about $2.1 \mathrm{Mb}$ between D1Mit325 and D1Mit21 (Chr. 1 64901285-66992999 bp), containing 38 genes, of which 28 genes are known and 10 genes are novel [27]. The exons and exon-intron boundaries of 22 genes were sequenced and no mutation was found. Next targeted resequencing of repro4 mutant DNA in a region of Chr. 1 $(64,835,695-66,992,999 \mathrm{bp})$ was conducted. repro4 mutant DNA from this region was enriched using a custom NimbleGen capture array and sequencing was performed on Illumina Genome Analyzer 2. Bioinformatic analysis revealed no mutations within exons, consistent with our sequencing results. One mutation, in an intron of the Mtap2 (microtubule-associated protein 2) gene that is located between exons 2 and 3 in ENSMUST00000114015 and ENSMUST00000114017 transcripts, was confirmed by sequencing repro4 mutant and B6 tail DNA (Figure 5a, b). This was a T to A transversion (Figure 5a). Subsequently, expression of Mtap2 in WT and repro4 mutant testes was examined. There are 9 protein-coding transcripts for Mtap2 [27]. The gene expression assay used covered 5 Mtap2 transcripts: ENSMUST00000114015, ENSMUST00000024639, ENSMUST00000114017, ENSMUST00000114013, ENSMUST00000114018. The remaining protein coding transcripts were not analyzed because they are at least $110 \mathrm{~kb}$ distant from the mutation. Quantitative RT-PCR showed that the transcript level of Mtap2 was more than 50\% less in repro4 mutant testes than in heterozygote littermate controls at postnatal day 8 (Figure 5c). A western blot analysis was performed to determine if MTAP2 protein was present; there were no major differences between the wild type and the repro4 mutant (data not shown). The antibody used for this analysis detects numerous MAPs, but we do not know if it detects all isoforms expressed in testes, or whatever isoform(s) might be affected by the repro4 mutation. Nonetheless, this result suggests the possibility that, despite reduced Mtap2 transcript, the protein amounts may not be significantly reduced.

Figure 5. A mutation was identified in the Mtap2 gene in repro4 mutant DNA. The T to A transversion is marked by an open box (a). The mutation is located between exon 2 and exon 3 of Mtap2 (b). Quantitative RT-PCR was used to assess levels of Mtap2 transcript in P8 heterozygote and repro4 mutant testes (c).

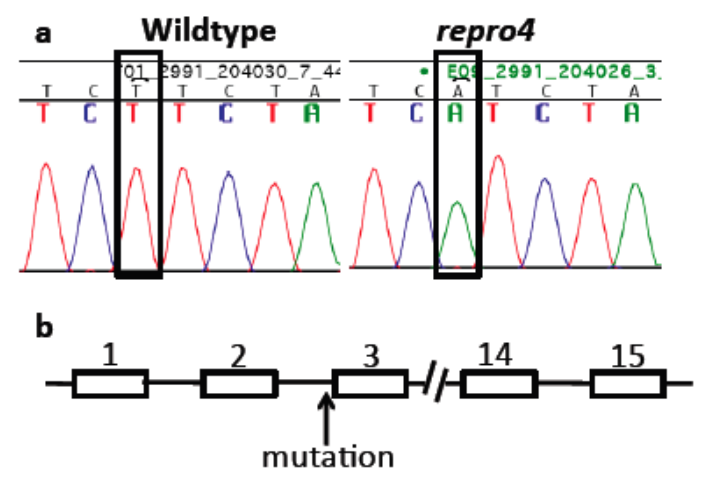

C

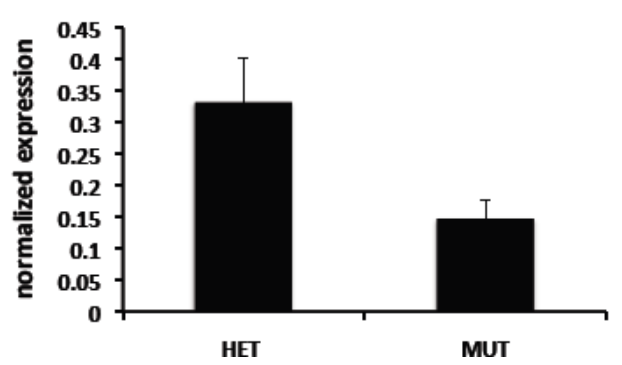


The Mtap2 locus in the mouse is complex, and up to 22 different mRNA transcripts can be produced. The transcripts differ by truncation of the 5'-end, truncation of the 3 '-end, presence or absence of 9 cassette exons, overlapping exons with different boundaries, alternative splicing or retention of one intron. Additionally, there are four probable alternative promoters. Of the predicted transcripts, 19 spliced and 2 unspliced mRNAs putatively encode a functional protein (20 different isoforms). The remaining mRNA variants (unspliced) appear not to encode protein [28]. Furthermore, $1025 \mathrm{bp}$ of this gene are antisense to putative spliced predicted gene Tahorora, raising the possibility of regulated alternative expression. Thus there is considerable molecular complexity, which may be reflected in biological complexity. In the literature, the encoded MTAP2 proteins are referred to as MAP2s (microtubule-associated protein 2). The MAP2 protein identified in humans and rats consists of alternatively spliced isoforms in two groups: high-molecular weight MAP2, including MAP2A and MAP2B, and low-molecular weight MAP2 including MAP2C and MAP2D with different spatiotemporal expression patterns in neurons [29-32]. Indeed, all studies of MAP2 are related to its function in neurons. However, the so-called "neuron-specific" MAP2 is also present both in somatic cells and germ cells of the rat testis $[15,16]$. The predominant MAP species in the rat testis is proposed to be the MAP2C protein. In addition to cytoplasmic localization, MAP2 was also found in nuclei of Sertoli cells, as well as pachytene and diplotene spermatocytes in rat testes, perhaps even co-localizing with the synaptonemal complex as MAP1B [17]. Based on these data, the mouse MTAP2 protein could be present in testes at the right time and in the right place to play roles in the meiotic prophase I to metaphase I transition. Our finding that a mutation in the Mtap2 gene in the mouse is associated with prophase arrest of meiosis I is consistent with this hypothesis.

How might the MTAP2 protein act in meiotic progress? MTAP2 is a microtubule stabilizing protein [29]. It is possible that the meiotic prophase arrest phenotype of repro4 mutants is a result of destabilization of microtubules, due to diminished Mtap2 expression. Indeed, the microtubule-destabilizing drug, colchicine, causes arrest of cell cycle both in mitosis and meiosis, as well as failed migration of elongated spermatids toward the tubule lumen and abnormal morphogenesis of the sperm head and flagellum [33-37]. Thus microtubule dynamics can be inferred to be an important regulatory mechanism in spermatogenesis. It has been demonstrated that MAPs can modulate the microtubule dynamics at extremely low concentrations [38], and possibly diminished, but not ablated, expression of Mtap2 could impact on testicular microtubule dynamics. If this is occurring in repro4 mutant testes, the implication is that microtubule dynamics may directly or indirectly influence meiotic progression from prophase I to the division phase, a previously unsuspected mechanism. More broadly, the involvement of MTAP2 in meiosis, implied by the repro4 mutant phenotype, is consistent with emerging evidence that motor proteins, which often use cytoskeleton for movement, are not only present in nuclei, but also play significant roles in chromosome condensation and segregation [39,40]. However, it is not known whether the meiotic arrest phenotype is a direct or indirect effect of the Mtap2 mutation. Thus, although this report provides novel data implicating Mtap2 in meiotic cell cycle regulation, more work is required to resolve the mechanism of the Mtap2 mutation in regulation of the meiotic G2/MI transition. 


\section{Experimental Section}

\subsection{Animals}

All mice were bred and raised in the research colony of the authors at The Jackson Laboratory. Protocols for their care and use were approved by the Institutional Animal Care and Use Committee (IACUC) of The Jackson Laboratory. All the repro4 mutant mice used for biological study were from a congenic line maintaining the repro4 mutation on a $\mathrm{C} 3 \mathrm{H}$ background.

\subsection{Mutagenesis, Genetic Fine Mapping and Sequencing}

Mutagenesis of adult male C57BL/6J (B6) mice were carried out with injection of ethylnitrosourea (ENU) as previously described [41]. The mice were subsequently crossed with $\mathrm{C} 3 \mathrm{HeB} / \mathrm{FeJ}(\mathrm{C} 3 \mathrm{H})$ females to create $\mathrm{G} 1$ male founders of pedigrees. G1 males were mated to $\mathrm{C} 3 \mathrm{H}$ females to produce $\mathrm{G} 2$ offspring. G2 daughters were backcrossed to the G1 father to generate the G3 population. The G3 offspring were tested for fertility phenotype [42].

DNA was extracted from a piece of tail of infertile G3 animals in the repro4 pedigree, and a genome scan was carried out by the Fine Mapping Service of The Jackson Laboratory using microsatellite markers polymorphic between $\mathrm{B} 6$ and $\mathrm{C} 3 \mathrm{H}$. Linkage analysis mapped the mutation to chromosome 1. For the genetic fine mapping, repro4 mutant females were crossed to strain CAST/EiJ males to take advantage of greater polymorphism. Genotyping was done by PCR amplification of tail DNA. The critical region was mapped to chromosome 1 between D1Mit325 and D1Mit21, about 2.1 Mb.

For sequencing, DNA was obtained from extraction of tails of repro4 mutant, repro 4 heterozygous and B6 mice. After PCR amplification, products were run on $2 \%$ SeaKem ${ }^{\circledR}$ LE Agarose gel (Lonza). Suitable bands were then cut and DNA was extracted using QIAquick ${ }^{\circledR}$ Gel Extraction Kit (Qiagen). DNA sequencing was performed using standard methods by the DNA Sequencing Service of The Jackson Laboratory. Only exons and intron-exon boundaries were sequenced.

For deep sequencing, DNA was isolated by the DNA Resource Service of The Jackson Laboratory. DNA samples were reextracted once with phenol-chloroform; the 260/280 ratio was about 2.0. DNA samples were enriched using a custom NimbleGen capture array and single lane sequencing on Illumina Genome Analyzer 2 was performed.

\subsection{Histology and Immunohistochemistry}

Testes were fixed in Bouin's or 4\% paraformaldehyde in PBS overnight and paraffin-embedded. $5 \mu \mathrm{m}$ sections were cut, dewaxed and stained with Periodic Acid Schiff (PAS). For immunohistochemistry, the sections were blocked with 5\% goat serum in PBS at room temperature for $30 \mathrm{~min}$. Primary antibodies were applied to the sections and incubated at $37{ }^{\circ} \mathrm{C}$ for $1 \mathrm{~h}$. The antibodies used included rabbit antibody to CDC2 (CDK1) (Santa Cruz) at 1:50 and mouse CCNB1 antibody (Abcam) at 1:400. After two washes with PBS, the color reactions were performed according to SuperPicTure $^{\mathrm{TM}}$ polymer detection Kit (Zymed). Briefly, the sections were incubated with HRP polymer conjugate (reagent A) at $37{ }^{\circ} \mathrm{C}$ for $10 \mathrm{~min}$, followed by two washes of PBS. AEC single 
solution chromogen (reagent B) was added to the sections, and the sections were then incubated at room temperature for $12 \mathrm{~min}$. After rinsing with distilled water, Hematoxylin (Sigma) was used for counterstaining. Clearmount (Zymed) was used as mounting solution. Images were acquired with a Leica DMRXE microscope coupled with a DFC 300FX R1 CCD camera.

\subsection{Germ Cell Isolation and Spermatocyte Enrichment}

Mice at suitable ages were killed by cervical dislocation. Testes were removed, detunicated and digested in $0.5 \mathrm{mg} / \mathrm{mL}$ collagenase (Sigma) in Krebs-Ringer bicarbonate solution (KRB) (120.1 mM NaCl, $4.8 \mathrm{mM} \mathrm{KCl}, 25.2 \mathrm{mM} \mathrm{NaHCO}_{3}, 1.2 \mathrm{mM} \mathrm{KH}_{2} \mathrm{PO}_{4}, 1.2 \mathrm{mM} \mathrm{MgSO}{ }_{4} \cdot 7 \mathrm{H}_{2} \mathrm{O}, 1.3 \mathrm{mM}$ $\mathrm{CaCl}_{2}, 11 \mathrm{mM}$ glucose, $1 \mathrm{X}$ essential amino acids, $1 \mathrm{X}$ nonessential amino acids) at $32{ }^{\circ} \mathrm{C}$ for $20 \mathrm{~min}$, followed by digestion in $0.5 \mathrm{mg} / \mathrm{mL}$ trypsin (Sigma) containing $20 \mathrm{mg} / \mathrm{mL}$ DNase I in KRB at $32{ }^{\circ} \mathrm{C}$ for $13 \mathrm{~min}$. After digestion the cell suspension was filtered through an $80-\mu \mathrm{m}$ mesh filter and washed three times in KRB. Germ cells were then processed as described below.

\subsection{Okadaic Acid-Induced Meiotic Prophase I to Metaphase I(G2/MI) Transition}

Enriched germ cells were placed in culture medium as previously reported [43] and incubated at $32{ }^{\circ} \mathrm{C}$ for 4 hours for recovery. The cells were then induced to undergo the G2/MI transition by the addition of OA (CalBiochem), dissolved at $300 \mu \mathrm{M}$ in ethanol and used at $4 \mu \mathrm{M}$ in the culture, while control cells were treated with the same volume of ethanol. After $4 \mathrm{~h}$ culture, the cells were collected for air-dried chromosome preparation.

\subsection{Surface-Spread Chromatin Preparation}

Spermatocytes were collected by centrifugation, surface-spread in wells of multi-spot microscope slides (Thermo) and fixed following the procedure previously described [44,45]. Prior to antibody labeling, slides were washed three times in washing/blocking buffer $(0.3 \% \mathrm{BSA}, 1 \%$ goat serum in phosphate-buffered saline, $\mathrm{pH} 7.4$ ); the second wash included $0.05 \%$ Triton-X 100. After draining, the slides were incubated overnight with primary antibodies. Primary antibodies used were rabbit anti-SYCP1 (Novus) at 1:100; rabbit anti-SYCP3 (Novus) at 1:100; guinea pig anti-H1t [5] at 1:500; rabbit anti- $\gamma \mathrm{H} 2 \mathrm{AX}$ (Millipore) at 1:200; rat anti-SYCP3 [46] at 1:1000; rabbit anti-ATR (Santa Cruz) at 1:100. Secondary antibodies against rabbit, rat, or guinea pig IgG and conjugated with Alexa 594 or 488 (Molecular Probes) were used at 1:500 dilution. Images were acquired with a Leica DMRXE epifluorescence microscope equipped with a 100X plan-neofluar oil-immersion objective lens and a triple filter (set no. 61000V2 BS\&M, Chroma Technology for simultaneous visualization of green (Alexa 488), red (Alexa 594), yellow (Alexa 488 + Alexa 594) and blue (DAPI) fluorescence). The microscope was linked to a Micromax cooled CCD camera (RS Princeton Instrument), a high-speed shutter driven by a Sutter Lambda 10-2 (Sutter Instrument) and Metamorph software (Universal Imaging Corporation) to capture the images.

Chromosome condensation was evaluated from air-dried chromosome preparations [47] from cultured spermatocytes collected by centrifugation and washed in $2.2 \%$ sodium citrate. The air-dried chromosome preparations were stained with Gurr Giemsa (Invitrogen). G2/MI stages, from pachynema 
to MI were scored using an Olympus microscope with a 40x plan objective and $10 \times$ ocular lenses, and images were captured to Adobe Photoshop with a Hamamatsu C5810 color-chilled camera (Photonic System).

\subsection{Western Blot Analysis}

For western blot analyses, total protein was extracted from testes of P21 mice using RIPA buffer (Santa Cruz) containing protease inhibitor cocktail (Santa Cruz). Protein concentration was measured by the BCA method. $10 \mu \mathrm{g}$ protein from each group was boiled for $3 \mathrm{~min}$, and proteins were separated by electrophoresis using 10\% SDS-PAGE. Proteins were transferred onto PVDF membrane (Millipore) based on the manufacturer's instruction. The blots were blocked with 5\% dried milk in Tris-buffered saline with $0.1 \%$ Tween-20 overnight at $4{ }^{\circ} \mathrm{C}$. Rabbit anti-CDC2 (CDK1) (Santa Cruz) at 1:200 and mouse anti-CCNB1 (Abcam) at 1:2000 were used. The blots were incubated for $2 \mathrm{~h}$ at room temperature, and then incubated with horseradish peroxidase-conjugated anti-IgG secondary antibodies made from mouse (Invitrogen) and rabbit (Invitrogen) for $1 \mathrm{~h}$ at room temperature. The proteins were detected using the ECL Plus Western Blotting Detection System (Amersham).

\subsection{Quantitative RT-PCR}

Total RNA was extracted from frozen testes of P8 mice using the RNeasy extraction kit with DNaseI treatment as described by the manufacturer (Qiagen). Samples were diluted to a concentration of $10 \mathrm{ng} / \mu \mathrm{L}$, dispensed in single-use aliquots, and stored at $-80{ }^{\circ} \mathrm{C}$. cDNA was made by using QuantiTech Reverse Transcription Kit (Qiagen) and followed the manufacturer's instruction. Quantitative RT-PCR (qRT-PCR) was performed on the Applied Biosystems 7500 Real-Time PCR System. TaqMan ${ }^{\circledR}$ gene expression assays Mm00485230_m1 for Mtap2 and Hs99999901_s1 for 18 s control (Applied Biosystems) were used to determine the relative expression level of Mtap2 according to the relative standard curve method.

\section{Conclusions}

The repro4 mutant phenotype of meiotic arrest is associated with a mutation in an intron of the Mtap2 gene, encoding a microtubule-associated protein. Although spermatocytes of repro4 mutants appear to execute key events of meiotic prophase I normally, the meiotic prophase I to metaphase I transition did not occur, thus causing sterility, and suggesting that mutation of MTAP2 might be associated also with cases of human infertility. The features of the repro4 mutant phenotype raise the intriguing possibility of a hitherto unsuspected regulatory pathway governing the progress of meiosis, a pathway dependent on stability of microtubules and perhaps also implicating other nuclear motor proteins in meiotic chromosome condensation and segregation. The Mtap2 gene and its encoded proteins exhibit considerable complexity and thus, further work should not only confirm mechanism for the effect of the detected mutation and the roles of MTAP2 protein in male germ cells, but also bring novel insights to meiotic chromosome dynamics. 


\section{Acknowledgements}

We appreciate the assistance of Heather Lothrop in maintaining mice, Sheila Bornstein and Janice Pendola for original identification of the repro4 mutant line, outstanding support from the Scientific Services of The Jackson Laboratory, members of the Handel Laboratory for discussion of this work, and John Eppig and Lindsay Shopland of The Jackson Laboratory for comments on the manuscript. We are indebted to John Schimenti (Cornell University) for frequent advice on gene identification strategies and to Benjamin King for analysis of sequence data. Generation of the repro4 mutant mice was supported by the NIH, HD42137, and this study was supported by NIH HD33816.

\section{References}

1. Handel, M.A.; Schimenti, J.C. Genetics of mammalian meiosis: Regulation, dynamics and impact on fertility. Nat. Rev. Genet. 2010, 11, 124-136.

2. Matzuk, M.M.; Lamb, D.J. Genetic dissection of mammalian fertility pathways. Nat. Cell Biol. 2002, 4, 41-49.

3. Matzuk, M.M.; Lamb, D.J. The biology of infertility: Research advances and clinical challenges. Nat. Med. 2008, 14, 1197-1213.

4. Yatsenko, A.N.; Iwamori, N.; Iwamori, T.; Matzuk, M.M. The power of mouse genetics to study spermatogenesis. J. Androl. 2010, 31, 34-44.

5. Cobb, J.; Cargile, B.; Handel, M.A. Acquisition of competence to condense metaphase I chromosomes during spermatogenesis. Dev. Biol. 1999, 205, 49-64.

6. Sun, F.; Handel, M.A. Regulation of the meiotic prophase I to metaphase I transition in mouse spermatocytes. Chromosoma 2008, 117, 471-485.

7. Sun, F.; Palmer, K.; Handel, M.A. Mutation of Eif4g3, encoding a eukaryotic translation initiation factor, causes male infertility and meiotic arrest of mouse spermatocytes. Development 2010, 137, 1699-1707.

8. Zhu, D.; Dix, D.J.; Eddy, E.M. HSP70-2 is required for CDC2 kinase activity in meiosis I of mouse spermatocytes. Development 1997, 124, 3007-3014.

9. Dix, D.J.; Allen, J.W.; Collins, B.W.; Mori, C.; Nakamura, N.; Poorman-Allen, P.; Goulding, E.H.; Eddy, E.M. Targeted gene disruption of Hsp70-2 results in failed meiosis, germ cell apoptosis, and male infertility. Proc. Natl. Acad. Sci. USA 1996, 93, 3264-3268.

10. Wiltshire, T.; Park, C.; Caldwell, K.A.; Handel, M.A. Induced premature G2/M-phase transition in pachytene spermatocytes includes events unique to meiosis. Dev. Biol. 1995, 169, 557-567.

11. Tarsounas, M.; Pearlman, R.E.; Moens, P.B. Meiotic activation of rat pachytene spermatocytes with okadaic acid: The behaviour of synaptonemal complex components SYN1/SCP1 and COR1/SCP3. J. Cell Sci. 1999, 112, 423-434.

12. Gavet, O.; Pines, J. Activation of cyclin B1-Cdk1 synchronizes events in the nucleus and the cytoplasm at mitosis. J. Cell Biol. 2010, 189, 247-259.

13. de Martino, C.; Capanna, E.; Nicotra, M.R.; Natali, P.G. Immunochemical localization of contractile proteins in mammalian meiotic chromosomes. Cell Tissue Res. 1980, 213, 159-178. 
14. Wang, Y.; Xing, M. Immunolocalization of actin in synaptonemal complexes in spermatocytes of Mesocricetus auratus. Yi Chuan Xue Bao 1999, 26, 150-156.

15. Loveland, K.L.; Hayes, T.M.; Meinhardt, A.; Zlatic, K.S.; Parvinen, M.; de Kretser, D.M.; McFarlane, J.R. Microtubule-associated protein-2 in the rat testis: A novel site of expression. Biol. Reprod. 1996, 54, 896-904.

16. Loveland, K.L.; Herszfeld, D.; Chu, B.; Rames, E.; Christy, E.; Briggs, L.J.; Shakri, R.; de Kretser, D.M.; Jans, D.A. Novel low molecular weight microtubule-associated protein-2 isoforms contain a functional nuclear localization sequence. J. Biol. Chem. 1999, 274, 19261-19268.

17. Gil-Alberdi, L.; del Mazo, J. Microtubule-associated proteins during mouse spermatogenesis: Localization of a protein immunologically related to brain MAP1B protein in the synaptonemal complex. Cytogenet. Cell Genet. 1992, 59, 1-5.

18. Ookata, K.; Hisanaga, S.; Okumura, E.; Kishimoto, T. Association of p34cdc2/cyclin B complex with microtubules in starfish oocytes. J. Cell Sci. 1993, 105, 873-881.

19. Shiina, N.; Moriguchi, T.; Ohta, K.; Gotoh, Y.; Nishida, E. Regulation of a major microtubuleassociated protein by MPF and MAP kinase. EMBO J. 1992, 11, 3977-3984.

20. Ward, J.O.; Reinholdt, L.G.; Hartford, S.A.; Wilson, L.A.; Munroe, R.J.; Schimenti, K.J.; Libby, B.J.; O'Brien, M.; Pendola, J.K.; Eppig, J.; Schimenti, J.C. Toward the genetics of mammalian reproduction: Induction and mapping of gametogenesis mutants in mice. Biol. Reprod. 2003, 69, 1615-1625.

21. Schimenti, J.; Bucan, M. Functional genomics in the mouse: Phenotype-based mutagenesis screens. Genome Res. 1998, 8, 698-710.

22. Handel, M.A.; Lessard, C.; Reinholdt, L.; Schimenti, J.; Eppig, J.J. Mutagenesis as an unbiased approach to identify novel contraceptive targets. Mol. Cell Endocrinol. 2006, 250, 201-205.

23. Enders, G.C.; May, J.J., П. Developmentally regulated expression of a mouse germ cell nuclear antigen examined from embryonic day 11 to adult in male and female mice. Dev. Biol. 1994, $163,331-340$.

24. Sancar, A.; Lindsey-Boltz, L.A.; Unsal-Kacmaz, K.; Linn, S. Molecular mechanisms of mammalian DNA repair and the DNA damage checkpoints. Annu. Rev. Biochem. 2004, 73, 39-85.

25. Handel, M.A.; Cobb, J.; Eaker, S. What are the spermatocyte's requirements for successful meiotic division? J. Exp. Zool. 1999, 285, 243-250.

26. Ohi, R.; Gould, K.L. Regulating the onset of mitosis. Curr. Opin. Cell Biol. 1999, 11 (2), 267-273.

27. Ensembl. Available on line: http://uswest.Ensembl.Org/mus_musculus/gene/summary? $\mathrm{G}=$ ensmusg00000015222;r=1:66221842-66489157 (accessed on 7 December 2010).

28. AceView: A comprehensive cDNA-supported gene and transcripts annotation. Genome Biol. 2006, 7, S12.

29. Poulain, F.E.; Sobel, A. The microtubule network and neuronal morphogenesis: Dynamic and coordinated orchestration through multiple players. Mol. Cell Neurosci. 2010, 43, 15-32.

30. Binder, L.I.; Frankfurter, A.; Kim, H.; Caceres, A.; Payne, M.R.; Rebhun, L.I. Heterogeneity of microtubule-associated protein 2 during rat brain development. Proc. Natl. Acad. Sci. USA 1984, $81,5613-5617$. 
31. Garner, C.C.; Brugg, B.; Matus, A. A 70-kilodalton microtubule-associated protein (MAP2c), related to MAP2. J. Neurochem. 1988, 50, 609-615.

32. Chung, W.J.; Kindler, S.; Seidenbecher, C.; Garner, C.C. MAP2a, an alternatively spliced variant of microtubule-associated protein 2. J. Neurochem. 1996, 66, 1273-1281.

33. Boekelheide, K.; Neely, M.D.; Sioussat, T.M. The Sertoli cell cytoskeleton: A target for toxicantinduced germ cell loss. Toxicol. Appl. Pharmacol. 1989, 101, 373-389.

34. Handel, M.A. Effects of colchicine on spermiogenesis in the mouse. J. Embryol. Exp. Morphol. 1979, 51, 73-83.

35. Russell, L.D.; Malone, J.P.; MacCurdy, D.S. Effect of the microtubule disrupting agents, colchicine and vinblastine, on seminiferous tubule structure in the rat. Tissue Cell 1981, 13, 349-367.

36. Tepperberg, J.H.; Moses, M.J.; Nath, J. Colchicine effects on meiosis in the male mouse. II. Inhibition of synapsis and induction of nondisjunction. Mutat. Res. 1999, 429, 93-105.

37. Tepperberg, J.H.; Moses, M.J.; Nath, J. Colchicine effects on meiosis in the male mouse. I. Meiotic prophase: Synaptic arrest, univalents, loss of damaged spermatocytes and a possible checkpoint at pachytene. Chromosoma 1997, 106, 183-192.

38. Wilson, L.; Panda, D.; Jordan, M.A. Modulation of microtubule dynamics by drugs: A paradigm for the actions of cellular regulators. Cell Struct. Funct. 1999, 24, 329-335.

39. Mazumdar, M.; Sundareshan, S.; Misteli, T. Human chromokinesin KIF4A functions in chromosome condensation and segregation. J. Cell Biol. 2004, 166, 613-620.

40. Mazumdar, M.; Misteli, T. Chromokinesins: Multitalented players in mitosis. Trends Cell Biol. 2005, 15, 349-355.

41. Wilson, L.; Ching, Y.H.; Farias, M.; Hartford, S.A.; Howell, G.; Shao, H.; Bucan, M.; Schimenti, J.C. Random mutagenesis of proximal mouse chromosome 5 uncovers predominantly embryonic lethal mutations. Genome Res. 2005, 15, 1095-1105.

42. Lessard, C.; Pendola, J.K.; Hartford, S.A.; Schimenti, J.C.; Handel, M.A.; Eppig, J.J. New mouse genetic models for human contraceptive development. Cytogenet. Genome Res. 2004, 105 (2-4), 222-227.

43. Handel, M.A.; Caldwell, K.A.; Wiltshire, T. Culture of pachytene spermatocytes for analysis of meiosis. Dev. Genet. 1995, 16, 128-139.

44. Cobb, J.; Miyaike, M.; Kikuchi, A.; Handel, M.A. Meiotic events at the centromeric heterochromatin: Histone $\mathrm{H} 3$ phosphorylation, topoisomerase II alpha localization and chromosome condensation. Chromosoma 1999, 108, 412-425.

45. Cobb, J.; Reddy, R.K.; Park, C.; Handel, M.A. Analysis of expression and function of topoisomerase I and II during meiosis in male mice. Mol. Reprod. Dev. 1997, 46, 489-498.

46. Eaker, S.; Pyle, A.; Cobb, J.; Handel, M.A. Evidence for meiotic spindle checkpoint from analysis of spermatocytes from Robertsonian-chromosome heterozygous mice. J. Cell Sci. 2001, 114, 2953-2965.

47. Evans, E.P.; Breckon, G.; Ford, C.E. An air-drying method for meiotic preparations from mammalian testes. Cytogenetics 1964, 15, 289-294.

(C) 2011 by the authors; licensee MDPI, Basel, Switzerland. This article is an open access article distributed under the terms and conditions of the Creative Commons Attribution license (http://creativecommons.org/licenses/by/3.0/). 